\title{
Functional connectivity alteration of the deprived auditory regions with cognitive networks in deaf and inattentive adolescents
}

Jianhong Li

Beijing Tongren Hospital

\section{Weiwei Men}

Peking University

Jia-Hong Gao

Peking University

Yang Wang

Medical College of Wisconsin

Xiaoxia Qu

Beijing Tongren Hospital

David Chao Dong Zhu

Michigan State University

Junfang Xian ( $\nabla$ cj.xianjunfang@vip.163.com )

Beijing Tongren Hospital https://orcid.org/0000-0003-2191-9393

\section{Research Article}

Keywords: attentional deficit, adolescents, deafness, functional connectivity, resting-state functional magnetic resonance imaging

Posted Date: November 17th, 2021

DOI: https://doi.org/10.21203/rs.3.rs-943954/v1

License: (c) (1) This work is licensed under a Creative Commons Attribution 4.0 International License. Read Full License

Version of Record: A version of this preprint was published at Brain Imaging and Behavior on February 26th, 2022. See the published version at https://doi.org/10.1007/s11682-022-00632-x. 


\section{Abstract}

Adolescents with early profound deafness may present with distractibility and inattentiveness. The brain mechanisms underlying these attention impairments remain unclear. We performed resting-state functional magnetic resonance imaging to investigate the functional connectivity of the superior temporal and transverse temporal gyri in 25 inattentive adolescents with bilateral prelingual profound deafness, and compared the results with those of 27 age-matched normal controls. Pearson and Spearman's rho correlation analyses were used to investigate the correlations of altered functional connectivity with the attention scores on the Montreal Cognitive Assessment, years of deafness, sign language, and hearing aid usage. Compared with normal controls, prelingual profound deafness demonstrated mainly decreased resting-state functional connectivity between the deprived auditory regions and several other brain functional networks, including the attention control, language comprehension, default-mode, and sensorimotor networks. Moreover, we also found enhanced restingstate functional connectivity between the deprived auditory cortex and salience network. These results indicate a negative impact of early hearing loss on the attentional and other high cognitive networks, and the use of sign language and hearing aids normalized the participants' connectivity between the primary auditory cortex and attention networks, which is crucial for the early intervention and clinical care of deaf adolescents.

\section{Introduction}

Hearing loss affects many aspects of an individual's life. Cognitive decline is commonly observed in deaf individuals, especially in profoundly deaf adolescents. This includes decline in language development (Cupples et al., 2018), reading comprehension (Wauters, van Bon, Tellings, \& van Leeuwe, 2006; Daza, Phillips-Silver, Ruiz-Cuadra, \& López-López, 2014), and executive function (Hall, Eigsti, Bortfeld, \& LilloMartin, 2018).

This evidence suggests that hearing loss may lead to cognitive impairment in profoundly deaf adolescents, which can be labeled as the deficit hypothesis. In contrast with the deficit hypothesis, previous performance test studies in deaf adults have shown that individuals with deafness try to cope with, or compensate for, the hearing loss by developing enhanced sensory abilities in other senses, such as improved peripheral visual motion detection and spatial positioning capability, which can be labeled as the compensatory hypothesis (Proksch \& Bavelier, 2002; Sladen et al., 2005; Sadato et al., 2005; Chen, Zhang, \& Zhou, 2006; Bavelier, Dye, \& Hauser, 2006; Dye, Baril, \& Bavelier, 2007; Dye, Green, \& Bavelier, 2009; Buckley, Codina, Bhardwaj, \& Pascalis, 2010; Codina, Buckley, Port, \& Pascalis, 2011). So far to date, there has been little agreement on whether early deafness leads to attentional deficits and/or compensatory enhancement. These two different views were attributed to the different types of performed test tasks and the ages of the participants enrolled in these studies. The studies that documented attentional deficits focused on deaf children while attentional enhancement was reported mostly in deaf adults. Attention represents a complicated cognitive process that depends on the content selected and the way that it is engaged. To reconcile these opposite views, Dye and Bavelier (2010) 
applied two visual-attention dynamic tests to assess deaf children and adults and found that deaf children did not always present deficits in all attentional tasks and exhibited normal attention by adulthood.

Moreover, sensory-deprived brain regions may change due to a functional loss and compensatory plasticity. Previous task-fMRI studies have indicated a reorganization of the auditory cortex, following early and profound deafness in adults with compensatory recruitment through visual, somatosensory, and even tactile processing (Auer, Bernstein, Sungkarat, \& Singh, 2007; Bavelier et al., 2006; Bavelier \& Neville, 2002; Finney, Fine, \& Dobkins, 2001; Karns, Dow, \& Neville, 2012; Levänen, Jousmäki, \& Hari, 1998; Neville \& Lawson, 1987). The planum temporale (Petitto et al., 2000; Sadato et al., 2005), superior temporal gyrus (STG) (Almeida et al., 2015; Ding et al., 2015; Karns et al., 2012; Li et al., 2013), and Hechl's gyrus (Karns et al., 2012; Meyer et al., 2007; Scott, Karns, Dow, Stevens, \& Neville, 2014; Smith et al., 2011) have been reported to be involved in auditory reorganization.

Recent resting-state functional magnetic resonance imaging (rs-fMRI) studies (mainly in deaf adults) have reported alterations of the resting-state functional connectivity (RSFC) between auditory and other primary sensory networks after auditory deprivation. An increased RSFC between the auditory cortex and the right middle temporal gyrus (MTG) was found in deaf older adults (Puschmann \& Thiel, 2017). Among 17 participants (age, 15-70 years) with unilateral sensorineural hearing loss, an increased RSFC was found between the non-deprived auditory cortical regions and both the visual (VIN) and somatosensory networks (Zhang et al., 2016). The effects of hearing deprivation on the functional reorganization of higher-level cognitive networks, such as the executive-control network (ECN) (Tibbetts et al., 2011), emotion-processing (Husain, Carpenter-Thompson, \& Schmidt, 2014), default-mode (DMN) (Zhang et al., 2016); Schmidt et al., 2013), language-control (LCN) (Liu et al., 2015) and salience networks (SAN) (Ding et al., 2016), have also been reported. However, these studies were based on a population of deaf adults or unilaterally deaf individuals. The effect of bilateral prelingual profound deafness (BPPD) on the FC between the deprived auditory cortical regions and higher-level networks in adolescents remains unknown. Resting-state $\mathrm{fMRI}$ studies on this specific population can provide insights on the mechanisms and timing of functional reorganization, and guidance on potential interventions.

Deafness can be perceived as a connectome disease (Kral, Kronenberger, Pisoni, \& O'Donoghue, 2016). Considering the auditory and cognitive-behavioral symptoms associated with deafness, it is reasonable to hypothesize that BPPD potentially affects functional interactions of the deprived auditory cortical regions with higher-level cognitive networks, such as the attention-control networks and DMN. The attention-control networks are neural circuits subserving attentional processing, which allow some items to be privileged while others are ignored (Astle \& Scerif, 2009). The DMN interacts with different networks to complete various cognitive processes (Gusnard, Akbudak, Shulman, \& Raichle, 2001; Fox et al., 2005; Leech, Kamourieh, Beckmann, \& Sharp, 2011). Previous fMRI studies in adults with bilateral or unilateral sensorineural hearing loss have also reported altered RSFC in the DMN. However, these results remain inconsistent (Husain et al., 2014; Schmithorst, Plante, \& Holland, 2014; Wang et al., 2014; Zhang et al., 2016). An investigation of the RSFC in deaf adolescents with BPPD may fill this knowledge gap. 
We hypothesized that the RSFC of the deprived auditory cortical regions to the attention-control networks, DMN, and LCN may be mainly decreased in adolescents with BPPD. To verify this hypothesis, we chose the STG and the transverse temporal gyrus (TTG), as the regions of interest for a seed-based rs-fMRI analysis to assess the FC between attentional and other brain networks. The STG contains a part of the primary auditory cortex that is directly affected by deafness. Prior studies have revealed cross-model plasticity in the STG following deafness. (Auer et al., 2007; Finney et al., 2001; Finney, Clementz, Hickok, \& Dobkins, 2003; Levänen et al., 1998) TTG, which is also referred to as Heschl's gyri, belongs to the primary auditory cortex. It is known to be the first cortical structure involved in processing incoming auditory information (Wong et al., 2008).

In an additional analysis, we investigated the potential association between RSFC alterations and deafness duration and the attention scores on the Montreal Cognitive Assessment to understand how deafness progressively affects normal brain network development. We also explored the potential correlation between RSFC alterations and current common interventions for deafness (sign language and hearing aid use) to understand how these interventions affect brain function, thus providing more evidence for the choice of intervention treatment method and timing of the intervention for deaf individuals.

\section{Materials And Methods}

\subsection{Participants}

Twenty-five adolescents with BPPD were recruited in this study, 11 of whom were congenitally deaf and 14 became deaf at a very early age ( $<6$ months) because of ototoxic drug ingestion. We also recruited 27 age-, sex-, and education-matched controls with normal subjects. Two deaf and four control individuals were excluded because of motion artifacts or incomplete MRI examinations, resulting in a final sample size of 46 participants. The hearing level of each participant was measured based on the pure-tone average, which was calculated by averaging the minimal sound level (measured by standard pure-tone audiometry in decibels [dB]) the participant could hear at frequencies of $0.25,0.5,1.0,2.0$, and $4.0 \mathrm{kHz}$. All deaf participants could only hear sounds above $90 \mathrm{~dB}$ through their better ear. All normal control participants could hear sounds $\leq 30 \mathrm{~dB}$ through both ears. The duration of deafness, hearing aid usage, and sign language usage were documented during interviews with the deaf participants (Table 1).

All participants had normal or corrected-to-normal vision. They were not taking psychoactive medication and had no neurological disease or other developmental abnormalities besides deafness; all were righthanded high-school students and their parents had normal hearing. All participants and their parents signed the written informed consent. This study was approved by the local Ethics Committee of Beijing Tongren Hospital (approval number, TRECKY2017-017; date, 2017-7-14).

\subsection{Cognitive function tests}


To assess cognitive function, all participants completed two 10-min cognitive tests: the Mini-mental State Examination (MMSE) and Montreal Cognitive Assessment (MoCA). For deaf participants, both cognitive tests were administered via sign language and lip-reading by their teacher, who was a sign-language expert. The MMSE and MoCA scores ranged between 0 and 30, with MMSE and MoCA scores $\geq 25$ and $\geq$ 26 , respectively, being considered as normal.

\subsection{Experimental design}

The participants, after undergoing a high-resolution T1-weighted anatomical imaging and a field map scan examination, were instructed to look at a white fixation cross with a black background on a projector screen but were instructed not to stare or strain their eyes. To monitor head motion and whether the participants' eyes were shut during the scanning session, a real-time monitoring system was employed in this study. An MR compatible camera was fixed at the back of a semi-transparent and semi-reflective mirror which was fixed on the head coil. A computer in the scanning room displayed the video of one of the participants' eyes and the plots of head movement in real-time (MR multi-parameter monitoring system, MD-M100, Shenzhen Sinorad Co., Shenzhen, China, http://www.sinorad.com/). Following the resting-state scanning, an additional high-resolution T2-weighted structure image was acquired. During the scanning process, the participants' hearing was protected; each participant was wearing $3 \mathrm{M}$ earplugs (model 1100; 3M Corp., Saint Paul, MN, USA), and two thick sponges were used to isolate the coil from the ears, fixing the participant's head and limiting noise to a certain extent.

\subsection{MRI data acquisition}

All participants were scanned using a Siemens Prisma 3T MR scanner (Siemens AG, Munich, Germany) with a 64-channel head-neck coil at the Center for MRI Research, Peking University. Each participant was asked to lie comfortably on the scanner's bed while their head was snugly fixed in place by two foam pads to minimize head movement. A high-resolution anatomical scan was acquired using a 3D Magnetization-Prepared RApid Gradient-Echo (MPRAGE) sequence with the following parameters: 192 continuous sagittal slices; repetition time $(T R)=2530 \mathrm{~ms}$; echo time $(\mathrm{TE})=2.98 \mathrm{~ms}$; inversion time $(\mathrm{TI})=$ $1100 \mathrm{~ms}$; flip angle $(\mathrm{FA})=7^{\circ}$; field of view $(\mathrm{FOV})=256 \mathrm{~mm}(\mathrm{FH}) \times 224 \mathrm{~mm}(\mathrm{AP})$; matrix $=256 \times 224$; slice thickness $=1 \mathrm{~mm}$; voxel size $=1 \mathrm{~mm} \times 1 \mathrm{~mm} \times 1 \mathrm{~mm}$, isotropic, interpolated to $0.5 \mathrm{~mm} \times 0.5 \mathrm{~mm} \times 1 \mathrm{~mm}$; bandwidth, $240 \mathrm{~Hz} /$ pixel; and time of acquisition $=5 \mathrm{~min} 58 \mathrm{~s}$. Before the resting-state acquisition, a gradient-echo field mapping scan was acquired to correct the distortion of echo planar images. The slice planes were scanned along the rectal gyrus with the following parameters: TR $=620 \mathrm{~ms}$; TE1 $/ \mathrm{TE2}=$ 4.92/7.38 ms; FA $=60^{\circ} ; \mathrm{FOV}=224 \mathrm{~mm} \times 224 \mathrm{~mm}$; matrix $=112 \times 112 ;$ slice thickness $=2 \mathrm{~mm} ; \mathrm{gap}=0.3$ $\mathrm{mm}$; voxel size $=2 \mathrm{~mm} \times 2 \mathrm{~mm} \times(2+0.3) \mathrm{mm}$; bandwidth $=565 \mathrm{~Hz} /$ pixel; the phase-encoding direction was from posterior to anterior; and time of acquisition $=2 \min 22 \mathrm{~s}$. Regarding high-resolution restingstate imaging, a Siemens Simultaneous Multi-Slice Echo Planar Imaging sequence was employed, and the location, orientation of FOV, and the phase-encoding direction were copied to those of the field map sequence with the following parameters: $\mathrm{TR}=2000 \mathrm{~ms} ; \mathrm{TE}=30 \mathrm{~ms} ; \mathrm{FA}=90^{\circ} ; \mathrm{FOV}=224 \mathrm{~mm} \times 224 \mathrm{~mm}$; matrix $=112 \times 112$; slice thickness $=2 \mathrm{~mm}$; gap $=0.3 \mathrm{~mm}$; voxel size $=2 \mathrm{~mm} \times 2 \mathrm{~mm} \times(2+0.3) \mathrm{mm} ; 62$ 
slices; acceleration factor in the phase-encoding direction $=2$; number of reference lines in the phaseencoding direction $=32$; slice acceleration factor $=2$; bandwidth $=2232 \mathrm{~Hz} /$ pixel; and time of acquisition, $8 \mathrm{~min} 13 \mathrm{~s}$. During the resting-state scanning, the physiological signals of pulse and respiration were acquired using the Siemens pulse and respiratory unit. Both B0 shim modes of field map and Siemens Simultaneous Multi-Slice Echo Planar Imaging (SMS-EPI) sequence were set to "ADVANCED," to ensure good B0 homogeneity to further reduce the distortion in the functional scans. Following the resting-state imaging, an additional 3D high-resolution T2-weighted scan was acquired, which was used to accurately segment the brain tissues combined with the T1 image in SPM12 (Statistical Parametric Mapping, Wellcome Department of Cognitive Neurology, London, UK) with the following parameters: 192 continuous sagittal slices; TR = $3200 \mathrm{~ms} ; \mathrm{TE}=408 \mathrm{~ms}$; FOV = $256 \mathrm{~mm}(\mathrm{FH}) \times 224 \mathrm{~mm}(\mathrm{AP}) ;$ matrix = 256 $\times 224$; acceleration factor in the phase-encoding direction $=2 ; 3 \mathrm{D}$ acceleration factor $=2$; numbers of reference lines in the phase-encoding direction and 3D were both 32; slice thickness $=1 \mathrm{~mm}$; voxel size $=$ $1 \mathrm{~mm} \times 1 \mathrm{~mm} \times 1 \mathrm{~mm}$; interpolated to $0.5 \mathrm{~mm} \times 0.5 \mathrm{~mm} \times 1 \mathrm{~mm}$; bandwidth $=723 \mathrm{~Hz} /$ pixel; and time of acquisition $=4 \mathrm{~min} 59 \mathrm{~s}$. All participants were asked to close their eyes during T1 and T2 high-resolution structure scan acquisition to reduce the effect of movement.

\subsection{Generation of seed regions for functional connectivity analyses}

The labels of the Anatomical Automatic Labeling template were not well-matched with the anatomical space of the Montreal Neurological Institute (MNI), such as the region of Heschl_L_aal including gray matter (GM) and white matter (WM). Therefore, seed regions were generated by segmenting a modified ICBM152_2009C T1-weighted template using the FreeSurfer software (Martinos Center, Charlestown, MA, USA) (Fischl, 2012). The procedure was carried out as follows: first, the ICBM152_2009C T1-weighted template was normalized to the MNI152 space using the new segmentation and normalizing tools of SPM12 (Statistical Parametric Mapping; http://www.fil.ion.ucl.ac.uk/spm/), as the spaces were slightly different between the ICBM152_2009C and MNI152. Subsequently, the masks of the TTG and STG on both brain hemispheres were acquired from the segmentation results (aparc+aseg.mgz) with FreeSurfer, using the modified ICBM152_2009C T1-weighted image (Supplementary Figure S1).

\subsection{Structural image preprocessing}

To refine the accuracy of the resting-state normalization data, a combined segmentation with T1- and T2weighted images was performed using SPM12 (Ashburner \& Friston, 2005). First, each participant's T2weighted images were co-registered to the T1-weighted images, and their coordinate origins $(0,0,0)$ were set to the center of the anterior commissure $(\mathrm{AC})$ manually; the $\mathrm{Y}$-axis passed through the points of the AC and posterior commissure (PC). Second, the adjusted T1- and T2-weighted images were resliced to the transverse position with the voxel size set as $1 \mathrm{~mm} \times 1 \mathrm{~mm} \times 1 \mathrm{~mm}$, and the uniformity of the image's intensity was corrected using ANTs N4 bias correction algorithm (Tustison et al., 2010). Third, the processed T1- and T2-weighted images were combined and segmented with the new segmentation tools of SPM12, capable of acquiring GM, WM, cerebrospinal fluid (CSF) images, and the deformation field file. 
Finally, the T2-weight brain image was generated by a brain mask, acquired from the masks of GM, WM, and CSF.

\subsection{Physiological data preprocessing}

Physiological noise affects the results of functional imaging (Hu et al., 1995), therefore, this was removed by physiological data logging and processing. A regression matrix was generated using the physiological noise data (Birn, 2012) which was used in the preprocessing phase of the resting-state data analysis. All physiological recording data were manually assessed to rule out the absence of recorded physiological signals or signal loss during recording. According to Hu's method (Hu et al., 1995), a second-order fitting method was used to process the physiological information of pulse and respiration. An $8 \star \mathrm{N}$ regression matrix of regression variables was obtained. Eight columns represented the second-order pulse amplitude, first-order pulse amplitude, second-order pulse phase, first-order pulse phase, second-order respiration amplitude, first-order respiration phase, second-order respiration phase, and first-order respiration phase fitting results. Next, the head movement variables and $8 * \mathrm{~N}$ columns of physiological regression matrices were combined into a regression matrix, after which the generated regression matrix was considered as a covariable in the preprocessing of the resting-state fMRI data to minimize the influence of head movement and physiological noise on the results.

\subsection{Resting-state fMRI data preprocessing}

Resting-state fMRI data were analyzed using SPM12 software. The first ten volumes of functional images were discarded for each participant to allow for steady-state magnetization. Subsequently, the data were corrected for slice timing. Corrected images were unwarped for distortion correction using field mapping images and the FieldMap toolbox in SPM12. Participants whose head motion exceeded $2 \mathrm{~mm}$ in translation and $0.6^{\circ}$ in rotation were excluded. The unwarped and realigned data were co-registered to the T2-weighted images and then normalized to the MNI space with the deformation field file and resampled to 2-mm isotropic voxels. The rest of the data were processed using the DPABI toolbox (Data Processing and Analysis for Brain Imaging) (14; http:// rfmri.org/dpabi) (Yan et al., 2016), which removed the linear tread of time courses, smoothed [ 55 5] and regressed out several nuisance signals (Friston 24 head motion parameters, global mean signal, WM and CSF signals, physiological noise) (Hu et al., 1995; Tustison et al., 2010), filtered low frequency $(0.01-0.1 \mathrm{~Hz})$ fluctuations, and calculated the whole-brain FC of the four seed regions (TTG and STG in both brain hemispheres).

\subsection{Statistical analyses for whole-brain functional connectivity}

A two-sample t-test was conducted for the whole brain functional connections of the four seed regions between two groups. $P<0.05$ false discovery rate and cluster $>100$ voxels were considered statistically significant (Supplementary Figures S2-S5).

\subsection{Correlation analysis}


In the statistical results from the functional connections of the four seed regions between groups, each cluster with significant differences was used as a mask to extract the average functional connection values in each mask area of each deaf participant, respectively. A correlation analysis was carried out between the extracted average functional connection values and clinical parameters. A Pearson's correlation analysis was performed to investigate the relationship between altered RSFC with the seed regions of interest and clinical parameters, including duration of hearing loss, sign language use, and hearing aid use (in years). Moreover, a Spearman's rho correlation analysis was performed to investigate the relationship between the RSFC alteration and score of the sign language. The multiple comparison correction of the $p$ values derived from correlations was performed using combined probability of Fisher method provided by a MATLAB toolbox named 'Multiple Testing Toolbox' (Víctor Martínez-Cagigal, 2021). As the deaf group demonstrated significantly lower scores on cognitive function tests, correlation analysis with clinical data was only performed among the deaf individuals, and a threshold $(P<0.05$, after correction) was used to reflect significance. A trend towards significant correlations $(P<0.05$, uncorrected) was also reported.

\section{Results}

\subsection{Participant demographics and clinical features}

Table 1 shows the demographics and clinical characteristics of the participants. As expected, the deaf group presented significantly lower MMSE and MoCA cognitive scores than the control group (two-tail $t$ test, all $P<0.001)$. Nevertheless, deaf participants still had normal cognitive scores. We further analyzed between-group differences at the sub-domains of MoCA (Table 2).

We found that the deaf group had significantly lower scores in the cognitive functions of language, attention, visuospatial/executive, abstraction, and delayed recall (two-tail $t$-test, all $P<0.05$ ). However, no significant differences in orientation function between the groups were observed (two-tail $t$-test, $P>0.05$ )

\subsection{RSFC analysis}

The brain regions which revealed significantly altered RSFC to the four seed regions (STG and TTG in both hemispheres) between the deaf and normal control groups were identified.

\subsubsection{RSFC alteration of the right STG}

Compared with normal controls, deaf participants showed significantly decreased RSFC with the right STG mainly in the right medial frontal gyrus (MeFG), left inferior temporal gyrus (ITG), right MTG, bilateral angular gyrus (AG), parahippocampal gyrus (PHC) postcentral gyrus (PoCG), right precentral gyrus (PreCG), posterior cingulate cortex (PCC), and right cerebellar posterior lobe (Post. Cb), which belong to the left frontoparietal network (LFP), DMN, LCN, sensorimotor network (SEN), attentional network (AN), and SAN. Conversely, deaf participants showed a significantly increased RSFC between the right STG and the bilateral insula (INS), middle frontal gyri (MFG), supramarginal gyrus (SMG), middle occipital gyrus 
(MOG), cerebellum, right median cingulate gyrus (DCG), left calcarine (CAL), and inferior parietal lobe (IPL). These regions mainly belong to the AN, SAN, DMN, and VIN (Table 3, Figure 1, and Supplementary Figure S2).

\subsubsection{RSFC alteration of the left STG}

Compared with normal controls, deaf participants showed significantly decreased RSFC with the left STG mainly in the bilateral PreCG, superior frontal gyrus (SFG), MTG, AG, PHC, MeFG, left ITG, and MFG, which belong to the SEN, LCN, LFP, DMN, and ECN. Conversely, deaf participants showed significantly increased RSFC between the left STG and the anterior cingulate cortex (ACC), bilateral INS, SMG, MFG, bilateral thalamus (THA), bilateral Post.Cb, right precuneus (PUCN), left superior parietal lobe (SPL), and left SFG. These regions mainly belong to the SAN, AN, LCN, and DMN (Table 3, Figure 1, Supplementary Figure S3).

\subsubsection{RSFC alteration of the right TTG}

Compared with normal controls, deaf participants revealed significantly decreased RSFC with the right TTG in the bilateral MTG, ITG, AG, inferior frontal gyri, IPL, left MeFG, MFG, left SFG, precuneus (PCUN), hippocampus (HIP), and DCG, which mainly belong to the LCN, DMN, ECN, and AN. Meanwhile, we also found significantly increased RSFC between the right TTG and left cerebellum 7b, right ACC, bilateral INS, left MFG, and right SMG. These regions belong to the SAN and AN (Table 4, Figure 1, Supplementary Figure S4).

\subsubsection{RSFC alteration of the left TTG}

Compared with normal controls, deaf participants showed significantly decreased RSFC with the left TTG in the bilateral MTG, AG, SFG, left MFG, and MeFG, which mainly belong to the $L C N, D M N, E C N$, and AN. Meanwhile, we also found significantly increased RSFC between the left TTG and the right ACC, bilateral INS, bilateral MFG, left Post. Cb, and right SMG. These regions mainly belong to the SAN and AN (Table 4, Figure 1, Supporting information, Figure S5).

\subsection{Correlation between RSFC alterations and clinical parameters}

A significant negative correlation of the average RSFC between the auditory networks and the nodes of the DMN and AN with the duration of deafness was found ( $P<0.05$, mt_fisher corrected) (Figure 2). However, we did not find any significant correlation between the alteration RSFC of the auditory networks and other clinical parameters, such as sign language and hearing aid experience. We only found a negative correlation trend between the duration of sign language and hearing aid use, sign language proficiency, and the alteration RSFC of the auditory networks with the nodes of the AN $(P<0.05$, uncorrected) (Figure 3).

\section{Discussion}


We investigated the impact of BPPD on brain function based on the RSFC of the auditory networks (with STG and TTG as the seed regions) in adolescents. We found weaker FC with the deprived auditory cortical regions in the DMN, LCN, ECN, and SEN, and enhanced RSFC between the deprived auditory cortical regions and SAN in participants with BPPD. Meanwhile, we found both decreased and increased RSFC between the deprived auditory cortical regions and AN in participants with BPPD.

To the best of our knowledge, this is the first study of adolescents, where significantly decreased and increased RSFC between the deprived auditory cortical regions and the nodes of the AN were observed, including the MeFG, ITG, SPL, median cingulate gyri, PreCG, cerebellar posterior lobe, and THA. As the hubs of the ventral attention networks, MeFG are involved in bottom-up triggered attention, while the PreCG, SPL, posterior cerebellum, and THA, the hubs of the dorsal attention system, are involved in the control of top-down controlled attention (Alavash, Tune, \& Obleser, 2019; Menon, 2011). The cingulate gyrus is important in the capture of biological- and cognitive-related events and subsequently participates in higher-level cognitive control tasks (Dosenbach, Fair, Cohen, Schlaggar, \& Petersen, 2008; Fox, Corbetta, Snyder, Vincent, \& Raichle, 2006; Fox et al., 2005; Levitin, \& Menon, 2008; Menon \& Uddin, 2010; Sridharan). The ITG is one of the hubs of the visual processing and is associated with the representation of colors, faces, places and objects. It may also be involved in visual and delayed memories (Lafer-Sousa \& Conway, 2013). The decreased FC between the ITG and the deprived auditory cortex may represent the neural nature of delayed memory decline in the deaf participants in this study. Our findings are partially consistent with those of previous fMRI studies (Bonna et al., 2021; Ding et al., 2016) that reported increased FC between the auditory cortex and the AN in deaf adults. Conversely, in addition to increased RSFC, we found significantly decreased RSFC between the primary auditory cortex and the nodes of the AN, which could be attributed to the research population. We investigated the RSFC alterations in adolescents with BPPD, while previous studies enrolled early deaf adults (Bonna et al., 2021; Ding et al., 2016). Both impairment and compensation is likely happen during the early years of hearing deprivation. Functional reorganization may occur in the auditory cortex over a long period (Cardin et al., 2013; Finney et al., 2001). The enhanced FC between the deprived auditory cortex and the AN reported in early deaf adults mainly reflected adaptation and compensatory remodeling following the long-term auditory deprivation. The deaf adolescents in this study had a shorter deafness duration in general compared with adults. A gradual progression in both impairment and compensation could occur.

Several behavioral tests have pointed to an attentional deficit in profoundly deaf adolescents. Compared with their hearing peers, they are characterized by increased inattentiveness, distractibility and impulsiveness, weaker sustained attention, and a declined ability in selective attention and cognitive control (Conway, Pisoni, \& Kronenberger, 2009; Daza \& Phillips-Silver, 2013; Horn, Davis, Pisoni, \& Miyamoto, 2005; Mitchell \& Quittner, 1996; Quittner, Smith, Osberger, Mitchell, \& Katz, 1994; Yucel \& Derim, 2008). The finding that decreased RSFC was mainly found between the deprived auditory cortical regions and attention-control networks revealed in this study may contribute to the fundamental reasons of classroom misbehavior, impaired speech and language acquisition, and other cognitive issues in adolescents with profound deafness (Kuppler, Lewis, \& Evans, 2013; Lieu, Tye-Murray, \& Fu, 2012; Tibbetts et al., 2011). 
This hypothesis is supported by the significantly lower overall MMSE and MoCA scores, and the score in the attention domain in the deaf group. Meanwhile, the increased RSFC between the auditory cortex and the MeFG, a node in the AN, probably reflects a compensatory effect. This interpretation is supported by normal MMSE and nearly normal MoCA scores in deaf participants.

Decreased functional connections were also found between the deprived auditory cortical regions and nodes of the DMN in the deaf group. The DMN is well known as a task-negative network (Fox et al., 2005). However, its nodes in the PCC, AG, PUCN, HIP, and mPFC have been reported to be crucial regarding high-level cognitive functions, such as self-related social-cognitive processes (Binder, Desai, Graves, \& Conant, 2009), emotion regulation (Etkin, Egner, \& Kalisch, 2011) and memory (Vannini et al., 2011). Several recent studies have reported that DMN abnormalities exist in various mental and neurological disorders, such as dementia, schizophrenia, epilepsy, depression, and autism (Kennedy, Redcay, \& Courchesne, 2006; Greicius, Srivastava, Reiss, \& Menon, 2004; Rangel, Camerer, \& Montague, 2008). Similarly, this study identified abnormal FC in adolescents with BPPD. The deficits were most prominent between the nodes of the AG, PCC, PUCN, and HIP in the DMN and auditory cortex; additionally, the precuneus showed significantly enhanced connectivity with the STG. The precuneus is one of the key nodes in the DMN and is also related to short-term memory recall (Cabeza \& Nyberg, 2000). This dissociation may be correlated with different aspects of cognitive dysfunction following hearing loss. Our results are consistent with those of previous rs-fMRI studies in deaf adults, which showed that the FC within or to the DMN was found to be altered (Schmithorst et al., 2014; Wang et al., 2014; Zhang et al., 2016). In line with these studies, the weaker FC between the DMN and the deprived auditory cortical regions may suggest that the $\mathrm{DMN}$ is involved in the network integration of sensory defects.

We also found increased connectivity of the deprived auditory cortical regions with the key nodes of the SAN in deaf adolescents, including the INS and the ACC. The SAN is engaged in integrating, detecting and filtering related emotional information, feelings and autonomy (Seeley et al., 2007). One major function of the SAN is the identification of the most relevant stimulus among a variety of external and internal stimuli to direct a behavior (Menon \& Uddin, 2010). Previous studies have shown a dysfunctional SAN in deaf participants. For example, structural studies revealed structural reinforcement of the SAN in deaf individuals (Allen et al., 2008). Moreover, previous functional studies have indicated a stronger FC in deaf individuals between the SAN and the auditory cortex when dealing with visual working memory tasks (Bavelier et al., 2008; Ding et al., 2016). The hyperactivity of the anterior insula node of the SAN has been thought to be related to anxiety disorders (Stein, Simmons, Feinstein, \& Paulus, 2007). The enhanced FC of the insula with the deprived auditory cortical regions, revealed in this study, may be attributed to anxiety, which is a comorbid feature of hearing loss (Ibañez, Gleichgerrcht, \& Manes, 2010).

Moreover, we found significantly decreased RSFC between the MTG overlapping with the AG and deprived auditory regions, notably in the right STG. MTG is one of the key nodes in the self-perception network and involved in visual memory (Bonilha et al., 2017). AG is a key node in the DMN and also plays an important role in language comprehension and sentence reading (Seghier, 2013). In daily life, deaf individuals rely on visual information for communication, such as sign language and lip-reading, as 
auditory based language comprehension is impaired (Hallam \& Corney, 2014). This impairment would severely derail language development in adolescents with prelingual and profound deafness. A previous study has reported decreased speech and language skills in unilateral deaf children compared with their hearing peers (Hine, Thornton, Davis, \& Debener, 2008). The decreased RSFC between deprived auditory regions and the LCN in deaf adolescents may be the underpinning neurobiological mechanism contributing to the deficits in language comprehension following auditory deprivation.

Interestingly, we detected decreased RSFC between the right STG and the PoCG in deaf adolescents. This decreased FC between auditory deprived areas and the SEN may reflect a general disruption following the lack of auditory input. This finding was consistent with those of recent studies on deaf and blind individuals, which reported a very similar effect of the weakened FC between the SEN and deprived sensory cortices (Bonna et al., 2019; Butler, de la Rua, Ward-Able, \& Lomber, 2018; Liu et al., 2007). Bonna et al. (2019) interpreted this reduction in terms of the neural efficiency hypothesis. According to this hypothesis, there is less engagement of somatomotor areas during trained skills, such as the use of sign language and enhanced tactile processing, which are efficient and automated in deaf individuals.

In this study, no significant RSFC alterations within the deprived auditory cortical regions in the deaf group were observed, consistent with two previous studies on deaf adults (Husain et al., 2014; Wang et al., 2014). We only found a small cluster in the CAL and MOG of the VIN, which significantly increased RSFC with the auditory networks in the deaf group, in line with the findings of previous studies on deaf adults (Husain et al., 2014; Puschmann \& Thiel, 2017; Schmidt, Akrofi, Carpenter-Thompson, \& Husain, 2013). However, these findings were inconsistent with a study on congenital deaf adults (Li et al., 2013). The differences may be attributed to differences in the participants' age, as discussed in a review paper by Dye and Bavelier (2010). Our study enrolled adolescents with BPPD while they enrolled deaf adults. The different results of the FC between the deprived auditory cortical regions and the VIN in adolescents and adults suggested that the cross-model development between the auditory network and the VIN might progressively develop with deafness duration. This also suggested the importance of following our deaf group or a similar deaf group of adolescents for more years to understand the progressive cross-model development.

A significant negative correlation was identified between the duration of deafness and the reduced functional connection of the auditory networks (TTG) with the nodes of the right MTG and IPL, which belong to the DMN and AN respectively. Moreover, we also found a negative correlation trend between the reduced functional connection of the auditory networks with the AN and the sign language duration and level, hearing aid use duration compared with the normal controls. This finding suggests that the impairment of the AN was reduced over time, with an increase in the use and proficiency of sign language. Simply put, the hearing function normally develops after the usage of sign language. This implies that sign language and hearing aids should be used as early as possible as an intervention tool. Conversely, a longer duration of deafness may result in lesser RSFC reduction between the auditory networks and the AN, indicating the emergence of a compensation mechanism. 
In general, we found mainly weaker but enhanced RSFC with deprived auditory cortical regions in distinct brain regions, partially consistent with previous fMRI studies. The increase in perceptual ability may be related to the lack of concentration, and the increased sensitivity to other sensory modes which may lead to distraction, especially at an earlier age.

This study had three main limitations. First, the sample size was relatively small. A further study using a larger sample size should be carried out in order to analyze the FC alterations in deaf children and inattentiveness in adolescents. Second, we focused on changes in FC in cognition-related brain networks in deaf participants, so we did not assess other behaviors of the deaf participants when we collected the data. The results revealed an altered RSFC in other networks beyond the cognitive brain networks between the groups. However, we could not analyze the relationship between these RSFC and the relevant behaviors. Therefore, we will add these data in the following studies. Third, the study was cross-sectional, not longitudinal. We intend to perform follow-up examinations of the same participants for a long period in the future.

\section{Conclusions}

We investigated the effects of bilateral prelingual profound auditory deprivation in adolescents on RSFC between the auditory network and other brain functional networks. The results revealed a decreased RSFC of the deprived auditory cortical regions with the attentional-control networks, DMN, LCN, and SEN, and enhanced RSFC between the deprived auditory cortical regions and the SAN in participants with BPPD, which indicated the direct negative impact of early auditory deprivation on high cognitive-related networks.

Moreover, the usage of sign language and hearing aids seemed to normalize the connectivity between the deprived auditory cortical regions and attention-control networks. The RSFC within and between the auditory network and the VIN seemed intact in adolescents with BPPD. A neuroimaging study with a long follow-up examination focused on deaf participants or a similar population may help us further shed light on the progressive cross-model development.

\section{Declarations}

\section{Acknowledgements}

We thank Lihua Tai, Xiaocui Wang, and Xinxing Fu for their assistance in participants' recruitment and hearing testing. We thank Mrs. Chunfang Yan in MRI scanning support. We also thank the National Center for Protein Sciences at Peking University in Beijing, China, for assistance with the MRI data acquisition and data analysis. This study was supported by National Natural Science Foundation of China [grant number, 81501449], Major Scientific Research Instrument Projects of the National Natural Science Foundation of China [grant number, 81627901], and the Beijing Brain Initiative of Beijing Municipal Science \& Technology Commission [grant number, Z181100001518003]. The funding sources had no role 
in the study design; in the collection, analysis, and interpretation of data; in the writing of the report; or in the decision to submit the article for publication.

\section{Funding}

This study was supported by National Natural Science Foundation of China [grant number, 81501449], Major Scientific Research Instrument Projects of the National Natural Science Foundation of China [grant number, 81627901], and the Beijing Brain Initiative of Beijing Municipal Science \& Technology Commission [grant number, Z181100001518003]. The funders had no role in the study design; in the collection, analysis or interpretation of data; in the writing of the report; or in the decision to submit the article for publication.

\section{Conflict of interest}

The authors report no financial relationships with commercial interests.

\section{Ethics approval}

This study was approved by the local Ethics Committee of Beijing Tongren Hospital (approval number, TRECKY2017-017; date, 2017-7-14).

\section{Consent to participate}

Informed consent was obtained from all individual participants and their parents included in the study.

\section{Consent to publication}

The authors agree to publication in the Journal of Brain Imaging and Behavior.

\section{Availability of data and materials}

The data that support the findings of this study are available on request from the corresponding author. The data are not publicly available due to privacy or ethical restrictions.

\section{Code availability}

Not applicable.

\section{Author contributions}

Conceptualization, Jianhong Li, Junfang Xian; Investigation, Jianhong Li, Weiwei Men, Xiaoxia Qu; Formal Analysis, Weiwei Men, Xiaoxia Qu; Data curation, Weiwei Men, Jianhong Li; Writing - Original Draft, Jianhong Li, Weiwei Men; Writing - Review \& Editing, Jianhong Li; Supervision, Junfang Xian, David Chao Dong Zhu, Jia-Hong Gao, Wang Yang. 


\section{References}

Alavash, M., Tune, S., Obleser, J., 2019. Modular reconfiguration of an auditory control brain network supports adaptive listening behavior. PNAS USA. 116, 660-

669. https://doi.org/10.1073/pnas.1815321116

Allen, J.S., et al., 2008. Morphology of the insula in relation to hearing status and sign language experience. Neuroscience. 12, 11900-11905. https://doi: 10.1523/JNEUROSCI.3141-08.2008.

Almeida, J., et al., 2015. Decoding visual location from neural patterns in the auditory cortex of the congenitally deaf. Psychol. Sci. 26, 1771-1782. https://doi.org/10.1177/0956797615598970

Ashburner, J., Friston, K. J., 2005. Unified segmentation. Neurolmage. 26, 839-851. https://doi.org/10.1016/j.neuroimage.2005.02.018

Astle, D. E., Scerif, G., 2009. Using developmental cognitive neuroscience to study behavioral and attentional control. Dev. Psychobiol. 51, 107-118. https://doi.org/10.1002/dev.20350

Auer, E. T., Jr., et al., 2007. Vibrotactile activation of the auditory cortices in deaf versus hearing adults. NeuroReport. 18, 645-648. https://doi.org/10.1097/WNR.0b013e3280d943b9

Bavelier, D., Dye, M. W., Hauser, P. C., 2006. Do deaf individuals see better? Trends Cogn. Sci.10, 512-518. https://doi.org/10.1016/j.tics.2006.09.006

Bavelier, D., Neville, H. J., 2002. Cross-modal plasticity: where and how? Nat. Rev. Neurosci. 3, 443452. https://doi.org/10.1038/nrn848

Bavelier, D., et al., 2008. Ordered short-term memory differs in signers and speakers: implications for models of short-term memory. Cognition. 107, 433-459. http://doi: 10.1016/j.cognition.2007.10.012.

Binder, J. R., et al., 2009. Where is the semantic system? A critical review and meta-analysis of 120 functional neuro imaging studies. Cereb. Cortex. 19, 2767-2796. https://doi.org/10.1093/cercor/bhp055

Bonilha, L., et al., 2017. Temporal lobe networks supporting the comprehension of spoken words. Brain. 140, 2370-2380. https://doi.org/10.1093/brain/awx169

Bonna, K., et al., 2021. Early deafness leads to re-shaping of global functional connectivity beyond the auditory cortex. Brain Imaging Behav.15:1469-1482. https://doi.org/10.1007/s11682-020-00346-y

Birn, R.M., 2012. The role of physiological noise in resting-state functional connectivity. Neuroimage. 62 , 864-870. https://doi.org/10.1016/j.neuroimage.2012.01.016

Buckley, D., et al., 2010. Action video game players and deaf observers have larger Goldmann visual fields. Vis. Res. 50, 548-556. https://doi.org/10.1016/j.visres.2009.11.018 
Butler, B. E., et al., 2018. Cortical and thalamic connectivity to the second auditory cortex of the cat is resilient to the onset of deafness. Brain Struct Funct. 223, 819-835. https://doi.org/10.1007/s00429-0171523-y

Cabeza, R., Nyberg, L., 2000. Imaging cognition II: An empirical review of 275 PET and fMRI studies. J. Cogn. Neurosci. 12, 1-47. https://doi.org/10.1162/08989290051137585

Cardin, V., et al., 2013. Dissociating cognitive and sensory neural plasticity in human superior temporal cortex. Nat. Commun. 4, 1473. https://doi.org/10.1038/ncomms2463

Chen, Q., Zhang, M., Zhou, X., 2006. Effects of spatial distribution of attention during inhibition of return (IOR) on flanker interference in hearing and congenitally deaf people. Brain Res. 1109, 117-127. https://doi.org/10.1016/j.brainres.2006.06.043

Codina, C., et al., 2011. Deaf and hearing children: a comparison of peripheral vision development. Dev. Sci. 14, 725-737. https://doi.org/10.1111/j.1467-7687.2010.01017.x

Conway, C. M., Pisoni, D. B., Kronenberger, W. G., 2009.. The importance of sound for cognitive sequencing abilities: the auditory scaffolding hypothesis. Curr Dir Psychol Sci. 18, 275-279.

https://doi.org/10.1111/j.1467-8721.2009.01651.x

Cupples, L., et al., 2018. Language development in deaf or hard-of-hearing children with additional disabilities: type matters! J. Intellect. Disabil. 62, 532-543. https://doi.org/10.1111/jir.12493

Daza, M. T., Phillips-Silver, J., 2013. Development of attention networks in deaf children: support for the integrative hypothesis. Res Dev Disabil. 34, 2661-2668. https://doi.org/10.1016/j.ridd.2013.05.012

Daza, M. T., et al., 2014. Language skills and nonverbal cognitive processes associated with reading comprehension in deaf children. Res Dev Disabil. 35, 3526-3533.

https://doi.org/10.1016/j.ridd.2014.08.030

Ding, H., et al., 2016. Enhanced spontaneous functional connectivity of the superior temporal gyrus in early deafness. Sci. Rep. 6, 23239. https://doi.org/10.1038/srep23239

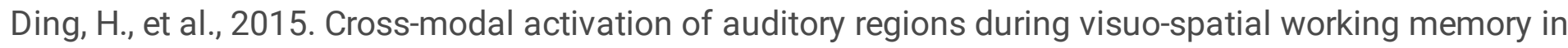
early deafness. Brain. 138, 2750-2765. https://doi.org/10.1093/brain/awv165

Dosenbach, N. U., et al., 2008. A dual-networks architecture of top-down control. Trends Cogn. Sci. 12, 99-105. https://doi.org/10.1016/j.tics.2008.01.001

Dye, M. W., Baril, D. E., Bavelier, D., 2007. Which aspects of visual attention are changed by deafness? The case of the Attentional Network Test. Neuropsychologia. 45, 1801-1811.

https://doi.org/10.1016/j.neuropsychologia.2006.12.019 
Dye, M. W., Bavelier, D., 2010. Attentional enhancements and deficits in deaf populations: an integrative review. Restor. Neurol. Neurosci. 28, 181-192. https://doi.org/10.3233/RNN-2010-0501

Dye, M. W., Green, C. S., Bavelier, D., 2009. The development of attention skills in action video game players. Neuropsychologia. 47, 1780-1789. https://doi.org/10.1016/j.neuropsychologia.2009.02.002

Etkin, A., Egner, T., Kalisch, R., 2011. Emotional processing in anterior cingulate and medial prefrontal cortex. Trends Cogn. Sci. 15, 85-93. https://doi.org/10.1016/j.tics.2010.11.004

Finney, E. M., et al., 2003. Visual stimuli activate auditory cortex in deaf participants: evidence from MEG. NeuroReport. 14, 1425-1427. https://doi.org/10.1097/00001756-200308060-00004

Finney, E. M., Fine, I., Dobkins, K. R., 2001. Visual stimuli activate auditory cortex in the deaf. Nat. Neurosci. 4, 1171-1173. https://doi.org/10.1038/nn763

Fischl, B., 2012. FreeSurfer. Neuroimage. 62, 774-781. http://doi: 10.1016/j.neuroimage.2012.01.021

Fox, M. D., et al., 2006. Spontaneous neuronal activity distinguishes human dorsal and ventral attention systems. PNAS USA. 103, 10046-10051. https://doi.org/10.1073/pnas.0604187103

Fox, M. D., et al., 2005. The human brain is intrinsically organized into dynamic, anticorrelated functional networks. PNAS USA. 102, 9673-9678. https://doi.org/10.1073/pnas.0504136102

Greicius, M. D., et al., 2004. Default-mode network activity distinguishes Alzheimer's disease from healthy aging: evidence from functional MRI. PNAS USA. 101, 4637-4642.

https://doi.org/10.1073/pnas.0308627101

Gusnard, D. A., et al., 2001. Medial prefrontal cortex and self-referential mental activity: relation to a default mode of brain function. PNAS USA. 98, 4259-4264. https://doi.org/10.1073/pnas.071043098

Hall, M. L., et al., 2018. Executive function in deaf children: auditory access and language access. J. Speech Lang. Hear. Res. 61, 1970-1988. https://doi.org/10.1044/2018_JSLHR-L-17-0281

Hallam, R. S., Corney, R., 2014. Conversation tactics in persons with normal hearing and hearingimpairment. Int. J. Audiol. 53, 174-181. https://doi.org/10.3109/14992027.2013.852256

Hine, J., et al., 2008. Does long-term unilateral deafness change auditory evoked potential asymmetries? Clin Neurophysiol. 119, 576-586. https://doi.org/10.1016/j.clinph.2007.11.010

Horn, D. L., et al., 2005. Development of visual attention skills in prelingually deaf children who use cochlear implants. Ear Hear. 26, 389-408. https://doi.org/10.1097/00003446-200508000-00003

$\mathrm{Hu}, \mathrm{X}$., et al., 1995. Retrospective estimation and correction of physiological fluctuation in functional MRI. Magn Reson Med. 34, 201-212. https://doi.org/10.1002/mrm.1910340211 
Husain, F. T., Carpenter-Thompson, J. R., Schmidt, S. A., 2014. The effect of mild-to-moderate hearing loss on auditory and emotion processing networks. Front. Syst. Neurosci. 8, 10. https://doi.org/10.3389/fnsys.2014.00010

Ibañez, A., Gleichgerrcht, E., Manes, F., 2010. Clinical effects of insular damage in humans. Brain Struct and Funct. 214, 397-410. https://doi.org/10.1007/s00429-010-0256-y

Karns, C. M., Dow, M. W., Neville, H. J., 2012. Altered cross-modal processing in the primary auditory cortex of congenitally deaf adults: a visual-somatosensory fMRI study with a double-flash illusion. J. Neurosci. 32, 9626-9638. https://doi.org/10.1523/JNEUROSCI.6488-11.2012

Kennedy, D. P., Redcay, E., Courchesne, E., 2006. Failing to deactivate: resting functional abnormalities in autism. PNAS USA. 103, 8275-8280. https://doi.org/10.1073/pnas.0600674103

Kral, A., et al., 2016. Neurocognitive factors in sensory restoration of early deafness: a connectome model. Lancet. Neurol. 15, 610-621. https://doi.org/10.1016/S1474-4422(16)00034-X

Kuppler, K., Lewis, M., Evans, A. K., 2013. A review of unilateral hearing loss and academic performance: is it time to reassess traditional dogmata? Int. J. Pediatr. Otorhinolaryngol. 77, 617-622. https://doi.org/10.1016/j.ijporl.2013.01.014

Lafer-Sousa, R., Conway, B. R., 2013. Parallel, multi-stage processing of colors, faces and shapes in macaque inferior temporal cortex. Nat. Neurosci. 16, 1870-1878. https://doi.org/10.1038/nn.3555

Leech, R., et al., 2011. Fractionating the default mode network: distinct contributions of the ventral and dorsal posterior cingulate cortex to cognitive control. J. Neurosci. 31, 3217-3224. https://doi.org/10.1523/JNEUROSCI.5626-10.2011

Levänen, S., Jousmäki, V., Hari, R., 1998. Vibration-induced auditory-cortex activation in a congenitally deaf adult. Curr. Biol. 8, 869-872. https://doi.org/10.1016/s0960-9822(07)00348-x

$\mathrm{Li}, \mathrm{Y}$., Booth, et al., 2013. Altered intra- and inter-regional synchronization of superior temporal cortex in deaf people. Cereb. Cortex. 23, 1988-1996. https://doi.org/10.1093/cercor/bhs185

Lieu, J. E., Tye-Murray, N., Fu, Q., 2012. Longitudinal study of children with unilateral hearing loss. Laryngoscope. 122, 2088-2095. https://doi.org/10.1002/lary.23454

Liu, B., et al., 2015. Functional connectivity in patients with sensorineural hearing loss using resting-state MRI. Am. J. Audiol. 24, 145-152. https://doi.org/10.1044/2015_AJA-13-0068

Liu, Y., et al., 2007. Whole brain functional connectivity in the early blind. Brain. 130, 2085-2096. https://doi.org/10.1093/brain/awm121 
Menon, V., 2011. Large-scale brain networks and psychopathology: a unifying triple network model. Trends Cogn. Sci. 15, 483-506. https://doi.org/10.1016/j.tics.2011.08.003

Menon, V., Uddin, L. Q., 2010. Saliency, switching, attention and control: a network model of insula function. Brain Struct. Funct. 214, 655-667. https://doi.org/10.1007/s00429-010-0262-0

Meyer, M., et al., 2007. Neuroplasticity of sign language: implications from structural and functional brain imaging. Restor. Neurol. Neurosci. 25, 335-351.

Mitchell, T. V., Quittner, A. L., 1996. Multimethod study of attention and behavior problems in hearingimpaired children. J. Clin. Child Psychol. 25, 83-96. https://doi.org/10.1207/s15374424jccp2501_10

Neville, H. J., Lawson, D., 1987. Attention to central and peripheral visual space in a movement detection task. III. Separate effects of auditory deprivation and acquisition of a visual language. Brain Res. 405, 284-294. https://doi.org/10.1016/0006-8993(87)90297-6

Petitto, L. A., et al., 2000. Speech-like cerebral activity in profoundly deaf people processing signed languages: implications for the neural basis of human language. PNAS USA. 97, 13961-13966. https://doi.org/10.1073/pnas.97.25.13961

Proksch, J., Bavelier, D., 2002. Changes in the spatial distribution of visual attention after early deafness. J. Cogn. Neurosci. 14, 687-701. https://doi.org/10.1162/08989290260138591

Puschmann, S., Thiel, C. M., 2017. Changed crossmodal functional connectivity in older adults with hearing loss. Cortex. 86, 109-122. https://doi.org/10.1016/j.cortex.2016.10.014

Quittner, A. L., et al., 1994. The impact of audition on the development of visual attention. Psychol. Sci. 5, 347-353. https://doi.org/10.1111/j.1467-9280.1994.tb00284.x

Rangel, A., Camerer, C., Montague, P. R., 2008. A framework for studying the neurobiology of value-based decision making. Nat. Rev. Neurosci. 9, 545-556. https://doi.org/10.1038/nrn2357

Sadato, N., et al., 2005. Cross-modal integration and plastic changes revealed by lip movement, randomdot motion and sign languages in the hearing and deaf. Cereb. Cortex. 15, 1113-1122. https://doi.org/10.1093/cercor/bhh210

Schmidt, S. A., et al., 2013. Default mode, dorsal attention and auditory resting state networks exhibit differential functional connectivity in tinnitus and hearing loss. PLOS ONE. 8, e76488. https://doi.org/10.1371/journal.pone.0076488

Schmithorst, V. J., Plante, E., Holland, S., 2014. Unilateral deafness in children affects development of multi-modal modulation and default mode networks. Front. Hum. Neurosci. 8, 164. https://doi.org/10.3389/fnhum.2014.00164 
Scott, G. D., et al., 2014. Enhanced peripheral visual processing in congenitally deaf humans is supported by multiple brain regions, including primary auditory cortex. Front. Hum. Neurosci. 8, 177. https://doi.org/10.3389/fnhum.2014.00177

Seeley, W. W., et al., 2007. Dissociable intrinsic connectivity networks for salience processing and executive control. J. Neurosci. 27, 2349-2356. https://doi.org/10.1523/JNEUROSCI.5587-06.2007

Seghier, M. L., 2013. The angular gyrus: multiple functions and multiple subdivisions. Neuroscientist. 19, 43-61. https://doi.org/10.1177/1073858412440596

Sladen, D. P., et al., 2005. Visual attention in deaf and normal hearing adults: effects of stimulus compatibility. J Speech Lang Hear Res. 48, 1529-1537. https://doi.org/10.1044/1092-4388(2005/106)

Smith, K. M., et al., 2011. Morphometric differences in the heschl's gyrus of hearing impaired and normal hearing infants. Cereb. Cortex. 21, 991-998. https://doi.org/10.1093/cercor/bhq164

Sridharan, D., et al., 2008. A critical role for the right fronto-insular cortex in switching between centralexecutive and default-mode networks. PNAS USA. 105, 12569-12574.

https://doi.org/10.1073/pnas.0800005105

Stein, M. B., et al., 2007. Increased amygdala and insula activation during emotion processing in anxietyprone subjects. Am. J. Psychiatry. 164, 318-327. https://doi.org/10.1176/ajp.2007.164.2.318

Tibbetts, K., et al., 2011. Interregional brain interactions in children with unilateral hearing loss. Otolaryngol. Head Neck Surg. 144, 602-611. https://doi.org/10.1177/0194599810394954

Tustison, N. J., et al., 2010. "N4ITK: improved N3 bias orrection". IEEE Trans Med Imaging. 29, $1310-$ 1320. http:// doi. org/10.1109/TMI.2010.2046908

Vannini, P., et al., 2011. What goes down must come up: role of the posteromedial cortices in encoding and retrieval. Cereb. Cortex. 21, 22-34. https://doi.org/10.1093/cercor/bhq051

Víctor Martínez-Cagigal (2021). Multiple Testing Toolbox (https://www.mathworks.com/matlabcentral/fileexchange/70604-multiple-testing-toolbox), MATLAB Central File Exchange. Retrieved October 21, 2021.

Wang, X., et al., 2014. Altered regional and circuit resting-state activity associated with unilateral hearing loss. PLOS ONE. 9, e96126. https://doi.org/10.1371/journal.pone.0096126

Wauters, L. N., et al., 2006. In search of factors in deaf and hearing children's reading comprehension. Am Ann Deaf. 151, 371-380. https://doi.org/10.1353/aad.2006.0041

Wong, P. C., et al., 2008. Volume of left Heschl's gyrus and linguistic pitch learning. Cereb Cortex. 18, 828836. https://doi.org/10.1093/cercor/bhm115 
Yan, C.G., et al., 2016. DPABI: Data Processing \& Analysis for (Resting-State) Brain Imaging. Neuroinformatics.14,339-351.

Yucel, E., Derim, D., 2008. The effect of implantation age on visual attention skills. Int. J. Pediatr. Otorhinolaryngol. 72, 869-877. https://doi.org/10.1016/j.ijporl.2008.02.017

Zhang, G. Y., et al., 2016. Changes of the directional brain networks related with brain plasticity in patients with long-term unilateral sensorineural hearing loss. Neuroscience. 313, 149161. https://doi.org/10.1016/j.neuroscience.2015.11.042

\section{Tables}

Table 1. Participants' demographics and clinical characteristics

\begin{tabular}{|llll|}
\hline & Deaf participants & Normal controls & $P$-value \\
\hline Sex (females/males) & $15 / 10$ & $15 / 12$ & 0.271 \\
\hline Age (years) & $15.75 \pm 0.84$ & $16.04 \pm 1.28$ & 0.342 \\
\hline Education level & $8.52 \pm 0.82$ & $8.47 \pm 1.81$ & 0.886 \\
\hline Right ear PTA (dB) & $100.30 \pm 9.00$ & $9.28 \pm 2.44$ & $<0.001$ \\
\hline Left ear PTA (dB) & $102.10 \pm 6.20$ & $9.44 \pm 2.67$ & $<0.001$ \\
\hline Age of onset of deafness (years) & $0.50 \pm 0.51$ & & \\
\hline Duration of deafness (years) & $15.00 \pm 1.10$ & & \\
\hline Duration of the sigh language use (years) & $6.72 \pm 2.76$ & & $<0.001$ \\
\hline Duration of the hearing aid use (years) & $7.06 \pm 4.04$ & & $<0.001$ \\
\hline MMSE scores & $28.16 \pm 1.28$ & $29.9 \pm 0.40$ & \\
\hline MoCA scores & $25.28 \pm 2.78$ & $29.9 \pm 0.30$ & \\
\hline
\end{tabular}

Abbreviations: dB, Decibel; MMSE, Mini-mental State Examination; MoCA, Montreal Cognitive Assessment; PTA, pure tone audiometry.

Table 2. Group differences of the cognitive domains in the Montreal Cognitive Assessment 


\begin{tabular}{|llll|}
\hline Sub-domains in MoCA & Deaf participants & Normal controls & $P$-value \\
\hline Visuospatial/Executive (5) * & $4.36 \pm 0.86$ & $5.00 \pm 0.00$ & 0.001 \\
\hline Naming (3) & $2.76 \pm 0.52$ & $3.00 \pm 0.00$ & 0.030 \\
\hline Attention (6) & $4.84 \pm 1.37$ & $6.00 \pm 0.00$ & $<0.001$ \\
\hline Language (3) & $1.84 \pm 0.94$ & $3.00 \pm 0.00$ & $<0.001$ \\
\hline Abstraction (2) & $1.20 \pm 0.92$ & $1.97 \pm 0.18$ & 0.003 \\
\hline Delayed recall (5) & $4.40 \pm 0.91$ & $4.97 \pm 0.18$ & 0.005 \\
\hline Orientation (6) & $5.88 \pm 0.33$ & $5.93 \pm 0.25$ & 0.510 \\
\hline Total score (30) & $25.28 \pm 2.78$ & $29.9 \pm 0.31$ & $<0.001$ \\
\hline
\end{tabular}

Abbreviation: MoCA, Montreal Cognitive Assessment.

*Maximum score is shown.

Table 3. Group differences in functional connectivity to the superior temporal gyrus 
Seed Cluster region $\operatorname{MNI}(x, y, z)$ Cluster size Peak $t$ value Network (\# of voxels)

\section{R STG Deaf $<$ Normal}

\begin{tabular}{|c|c|c|c|c|}
\hline R MeFG & $6,30,-14$ & 7859 & -7.73 & AN \\
\hline L ITG & $-62,-10,-26$ & 4093 & -8.04 & AN \\
\hline R MTG & $64,-30,-14$ & 3237 & -6.82 & LCN \\
\hline L AG & $-52,-60,24$ & 1666 & -6.32 & DMN \\
\hline PCC & $0,-48,20$ & 1570 & -5.95 & DMN \\
\hline R AG & $58,-62,30$ & 1126 & -5.89 & DMN \\
\hline L PoCG & $-52,-12,34$ & 658 & -5.70 & SEN \\
\hline R Cbe8 & $44,-74,-36$ & 600 & -5.71 & AN \\
\hline R PoCG & $58,-4,26$ & 536 & -4.99 & SEN \\
\hline R MeFG & $6,-24,56$ & 414 & -5.36 & AN \\
\hline L PHC & $-18,-14,-30$ & 241 & -5.84 & DMN \\
\hline R PreCG & $32,-22,58$ & 235 & -4.25 & SEN \\
\hline R PHC & $30,-18,-20$ & 105 & -3.98 & DMN \\
\hline \multicolumn{5}{|c|}{ Deaf > Normal } \\
\hline R DCG & $10,24,30$ & 3236 & 6.23 & AN \\
\hline R SMG & $60,-32,30$ & 3059 & 7.04 & LCN \\
\hline R INS & $48,10,-2$ & 3028 & 6.95 & SAN \\
\hline L Cbe8 & $-40,-54,-50$ & 2935 & 7.67 & AN \\
\hline L INS & $-14,-14,16$ & 1842 & 6.88 & SAN \\
\hline R MFG & $34,38,18$ & 856 & 5.89 & AN \\
\hline L MFG & $-42,44,24$ & 719 & 5.47 & AN \\
\hline L SPL & $-16,-68,40$ & 654 & 4.59 & AN \\
\hline R CbeCru1 & $40,-50,-32$ & 563 & 5.68 & CEN \\
\hline L SMG & $-60,-30,30$ & 511 & 4.85 & LCN \\
\hline L CAL & $-4,-78,8$ & 379 & 4.48 & VIN \\
\hline R MOG & $44,-74,20$ & 349 & 4.60 & AN \\
\hline
\end{tabular}




$\begin{array}{lllll}\text { L IPL } & -30,-48,48 & 313 & 4.70 & \text { DMN } \\ \text { L MOG } & -40,-78,22 & 158 & 4.93 & \text { AN } \\ \text { L ITG } & -44,-58,-14 & 125 & 4.39 & \text { DMN }\end{array}$

\section{STG Deaf $<$ Normal}

\begin{tabular}{lllll} 
L PoCG & $-54,-12,36$ & 694 & -5.88 & SEN \\
\hline R PreCG & $64,0,28$ & 640 & -6.06 & SEN \\
\hline R PreCG & $34,-14,70$ & 563 & -5.58 & SEN \\
\hline R MTG & $66,-10,-24$ & 327 & -4.49 & LCN \\
\hline L MeFGorb & $-4,42,-10$ & 322 & -5.41 & LFP \\
\hline R AG & $34,-68,54$ & 319 & -4.65 & DMN \\
\hline R SFG & $18,68,2$ & 182 & -4.78 & AN \\
\hline L SFG & $-22,38,50$ & 180 & -4.33 & AN \\
\hline L ITG & $-64,-40,-18$ & 158 & -5.97 & AN \\
\hline L MFG & $-34,20,56$ & 153 & -4.56 & AN \\
\hline R MeFG & $-2,16,-18$ & 113 & -4.17 & LFP \\
\hline L MTG & $-44,14,-24$ & 104 & -3.88 & LCN \\
\hline Deaf $>$ Normal & & & & \\
\hline R ACC & $2,28,24$ & 907 & 7.09 & SAN \\
\hline L Cbe8 & $-36,-62,-52$ & 545 & 6.85 & AN \\
\hline L INS & $-36,8,-2$ & 509 & 5.26 & SAN \\
\hline R Cbe8 & $34,-66,-54$ & 500 & 5.62 & AN \\
\hline R MFG & $32,38,20$ & 425 & 5.37 & AN \\
\hline L SMG & $-60,-32,32$ & 416 & 4.81 & LCN \\
\hline R SMG & $64,-36,30$ & 402 & 5.19 & LCN \\
\hline R INS & $46,14,2$ & 322 & 5.98 & SAN \\
\hline L THA & $-14,-14,16$ & 293 & 5.63 & AN \\
\hline L SPL & $-12,-70,40$ & 265 & 5.92 & AN \\
\hline L MFG & $-42,44,26$ & 184 & 5.55 & AN \\
\hline L SFG & $-12,4,72$ & 178 & 5.22 & AN \\
\hline & & & & \\
\hline
\end{tabular}




\begin{tabular}{lllll} 
R DCG & $12,-34,44$ & 167 & 5.80 & AN \\
\hline R PCUN & $18,-66,42$ & 142 & 4.71 & DMN \\
\hline L CbeCru1 & $-2,-78,-22$ & 138 & 5.12 & CEN \\
\hline R THA & $8,-18,16$ & 134 & 4.95 & AN
\end{tabular}

Note: Thresholds were set at $P \leq 0.05$ (corrected).

Abbreviations: $A C C$, anterior cingulate cortex; $A G$, angular gyrus; $A N$, attention control network; $C A L$, Calcarine; Cbe8, Cerebelum_8; CbeCru1, Cerebelum_Crus1; CEN, cerebellum network; DCG, median cingulate gyrus; DMN, default mode network; INS, insula; IPL, inferior parietal lobule; ITL, inferior temporal gyrus; ITG, inferior temple gyrus; L, left; LCN, language comprehension network; LFP, left frontoparietal network; MeFG, medial frontal gyrus; MFG, middle frontal gyrus; MNI , Montreal neurological institute; MOG, middle occipital gyrus; MTG, middle temporal gyrus; PCC, posterior cingulate cortex; PCUN, precuneus; PHC, parahippocampal gyrus; PoCG, postcentral gyrus; PreCG, precentral gyrus; R, right; SAN, salience network; SEN, sensorimotor network; SFG, superior frontal gyrus; SMG, supramarginal gyrus; SPL, superior parietal lobule; STG, superior temporal gyrus; THA, Thalamus; VIN, visual network.

Table 4. Group differences in functional connectivity to the transverse temporal gyrus 
Seed Cluster region $\operatorname{MNI}(x, y, z)$ Cluster size Peak $t$ value Networks (\# of voxels)

\section{R TTG Deaf $<$ Normal}

\begin{tabular}{|c|c|c|c|c|c|}
\hline & L MTG & $-60,-8,-24$ & 1295 & -8.25 & AN \\
\hline & R MTG & $62,-16,-22$ & 1217 & -7.69 & AN \\
\hline & L MeFGorb & $-4,46,-12$ & 1075 & -6.29 & AN \\
\hline & L AG & $-38,-70,48$ & 852 & -5.22 & DMN \\
\hline & R AG & $42,-62,36$ & 745 & -6.58 & DMN \\
\hline & L SFGmed & $-6,52,40$ & 436 & -6.16 & DMN \\
\hline & L IFGorb & $-46,30,-14$ & 253 & -5.86 & AN \\
\hline & R MFGorb & $34,40,-14$ & 206 & -5.57 & AN \\
\hline & L MFG & $-30,20,48$ & 157 & -4.85 & AN \\
\hline & L DCG & $-2,-36,34$ & 154 & -5.47 & AN \\
\hline & L PCUN & $-2,-54,20$ & 113 & -4.66 & DMN \\
\hline & L SFG & $-12,64,24$ & 106 & -5.39 & DMN \\
\hline & L HIP & $-26,-22,-14$ & 105 & -5.66 & DMN \\
\hline & \multicolumn{5}{|c|}{ Deaf $>$ Normal } \\
\hline & L Cbe7b & $-40,-56,-48$ & 546 & 7.55 & AN \\
\hline & R ACC & $4,30,22$ & 428 & 6.72 & SAN \\
\hline & R INS & $46,8,-2$ & 427 & 6.46 & SAN \\
\hline & L MFG & $-28,42,16$ & 249 & 5.66 & AN \\
\hline & R SMG & $64,-28,34$ & 170 & 5.03 & LCN \\
\hline & L INS & $-28,16,8$ & 116 & 5.65 & SAN \\
\hline \multirow[t]{6}{*}{ L TTG } & \multicolumn{5}{|c|}{ Deaf $<$ Normal } \\
\hline & R MTG & $62,-8,-24$ & 1042 & -7.18 & AN \\
\hline & $\mathrm{R} I P L / A G$ & $42,-62,38$ & 671 & -6.06 & DMN \\
\hline & L MTG & $-60,-2,-24$ & 641 & -7.38 & AN \\
\hline & L AG & $-40,-60,34$ & 578 & -5.05 & DMN \\
\hline & L SFGmed & $-10,50,44$ & 515 & -5.82 & DMN \\
\hline
\end{tabular}




\begin{tabular}{lllll} 
L MeFGorb & $-4,42,-10$ & 291 & -5.36 & SAN \\
\hline R MFG & $28,20,48$ & 183 & -5.45 & AN \\
\hline R SFGmed & $4,66,8$ & 162 & -4.91 & DMN \\
\hline Deaf $>$ Normal & & & & \\
\hline R ACC & $2,28,24$ & 415 & 6.47 & SAN \\
\hline R INS & $46,12,-4$ & 338 & 7.23 & SAN \\
\hline L INS & $-38,12,-2$ & 253 & 5.52 & SAN \\
\hline L MFG & $-34,46,22$ & 249 & 6.06 & AN \\
\hline L Cbe8 & $-38,-56,-48$ & 214 & 7.71 & AN \\
\hline R MFG & $32,44,28$ & 120 & 4.45 & AN \\
\hline R SMG & $68,-26,34$ & 112 & 5.02 & LCN
\end{tabular}

Note: Thresholds were set at $P \leq 0.05$ (corrected).

Abbreviations: ACC, anterior cingulate cortex; AG, angular gyrus; $\mathrm{AN}$, attention control network; Cbe7b, Cerebelum_7b; Cbe8, Cerebellum 8; DCG, median cingulate gyrus; DMN, default mode network; HIP, Hippocampus; IPL, inferior parietal lobule; ITG, inferior temporal gyrus; L, left; LCN, language comprehension network; MeFG, media frontal gyrus; MFG, middle frontal gyrus; MNI, Montreal Neurological Institute; MTG, middle temporal gyrus; PCUN, precuneus; R, right; SAN, salience network; SFGmed, superior frontal gyrus, medial; TTG, transverse temporal gyrus.

\section{Supplementary Files}

This is a list of supplementary files associated with this preprint. Click to download.

- Supplementarymaterials.docx 